\title{
Fully Crystallized Silicon Nanostructured Film Prepared at Low Temperatures by Plasma-Enhanced Chemical Vapor Deposition
}

\author{
Dmitry Evgenyevich Milovzorov \\ FLUENS Technology Group Ltd., Moscow, Russian Federation
}

Email address:

dmilovzorov2002@yahoo.com

\section{To cite this article:}

Dmitry Evgenyevich Milovzorov. Fully Crystallized Silicon Nanostructured Film Prepared at Low Temperatures by Plasma-Enhanced Chemical Vapor Deposition. American Journal of Nanosciences. Vol. 3, No. 3, 2017, pp. 39-46. doi: 10.11648/j.ajn.20170303.12

Received: May 14, 2017; Accepted: June 6, 2017; Published: July 14, 2017

\begin{abstract}
I studied the nanocrystalline silicon thin films by means of photoluminescent spectroscopy, Raman spectroscopy and Fourier-transformed infrared spectroscopy technique. The chemical bonding properties was studied by using the Fouriertransformed infrared spectroscopy in the range from $500 \mathrm{~cm}^{-1}$ to $2300 \mathrm{~cm}^{-1}$. Spectral peak at wave number of $1100 \mathrm{~cm}^{-1}$ is related to the Si-O-Si bonding configuration. Hydrogenation of film can be estimated by using spectral lines at $2100 \mathrm{~cm}^{-1}$ and $2080 \mathrm{~cm}^{-1}$. The Si-O dipoles which are located into silicon film play great role because of electron affinity for oxygen. Photoluminescent (PL) properties are significant for the films which were made by using hydrogenation of silicon. Fouriertransformed infrared spectra of film's absorption show the changes in chemicals in the film: from the oxygen incorporation into silicon to the elimination the Si-O bonding by adding the silicon tetra fluoride into electrochemical reactor, and increasing the $\mathrm{Si}-\mathrm{F}$ density of bonds. The dissociation of $\mathrm{SiF}_{4}$ molecule causes the appearance of fluorine on the film surface, but the hydrogen atoms react with fluorine and excess fluorine is removed. By increase of $\mathrm{SiF}_{4}$ flow rate, the $\mathrm{Si}-\mathrm{O}-\mathrm{Si}$ bridges were etched by HF acid. The size effect in PL from nanocrystalline silicon film can be explained by means of statistical method. We suggested that the $f(x)$ is distribution function of crystallites in their sizes, $\theta(x)$ is a quantum efficiency of nanocrystals. Accordingly, the quantity of emitted hydrogenizednanocrystals by band-to-band radiative transitions is important to estimate the PL linear optical response. The processing of grown hydrogeneited silicon nanocrystalline film by annealing and etching the oxides results in preparation of fully crystallized silicon film for manufacturingthe photonic devices with significant quantum yield.
\end{abstract}

Keywords: Photoluminescent Properties, Raman Spectra, Fourier-Transformed Infrared Spectra, Silicon Nanocrystals, Silicon Film Morphology

\section{Introduction}

The silicon film technology has the practical significance in electronic device manufacturing, such as thin film transistors, photonic devices, and integration circuits. The problem to eliminate the defects in silicon which change the electrical parameters by lowering of performance is very important. For this purpose the passivation by hydrogen of shallow single donors and acceptors can be done. There are numerous ways: hydrogen plasma processing the silicon surface, and hydrogen ion implantation or hydrogen gas annealing. All of them are to reduce the high defect density because the hydrogen atoms terminated the dangling bonds. In recent decade the hydrocarbon treated silicon surface was intensively studied $[1,2]$. Such exciting exploration is caused by need in removing of oxidized silicon layer from surface for further technological purposes, from one side, and to design the new electronic device with high performance, from another side. The hydrocarbons molecules chemisorbed on the silicon surface cause the alkyl chains production with hydrogenation of surface. The surface diffusion of hydrogen is sufficient even at low temperatures. Because, the high content of hydrogen atoms in hydrocarbon molecules can be transformed into hydrogen surface coverage. Accordingly, such various improvements in thin film transistor (TFT) technology can be caused to increase in $\mathrm{I}_{\text {on }} / \mathrm{I}_{\text {off }}$ ratio. By using plasma-enhanced chemical vapor deposition (PECVD) method for the film preparation at temperature of deposition 
around [3] 400-500 ${ }^{\circ} \mathrm{C}$ the following reactions can be occurred on surface: $2 \mathrm{SiH}_{2} \rightarrow \mathrm{Si}_{2} \mathrm{H}_{2}+\mathrm{H}_{2} ; \mathrm{Si}_{2} \mathrm{H}_{2} \rightarrow \mathrm{Si}_{2}+\mathrm{H}_{2}$. By using glass substrate and preliminary substrate processing by nitrogen plasma there are many particles of $(\mathrm{SiO})_{\mathrm{x}}$ and the roughness of substrate increases [4]. The nucleation process is critical to the density of precipitate $(\mathrm{SiO})_{\mathrm{x}}$ and depends on the preliminary processing of substrate. Because, the nucleifor crystal growth are appeared by the following reactions: $\mathrm{Si}-\mathrm{O}-\mathrm{Si}-\mathrm{O} \rightarrow \mathrm{Si}+\mathrm{SiO}_{2}$. By the way of hydrogeneition of silicon along with etching the nanocrystals and oxides by silicon fluorides, and removing molecular assemblies during the film growth I prepared the films with high performance.

\section{Silicon Films Preparation}

\subsection{Chemical Processes During Silicon Films Preparation}

I used low temperature conditions and high pressure of gas mixture to prepare the thin silicon film according to atomic layer deposition technological methods. However, the rate deposition was low, less than $5 \mathrm{~nm} / \mathrm{min}$. The chemical reactions on silicon surface by low temperature annealing $\left(300{ }^{\circ} \mathrm{C}\right)$ are following:

$$
\begin{gathered}
\mathrm{SiH}_{4} \rightarrow \mathrm{SiH}_{2}+\mathrm{H}_{2} ; \\
\mathrm{SiH}_{2} \rightarrow \mathrm{SiH}+\mathrm{SiH} ; \\
\mathrm{SiH}+\mathrm{SiH} \rightarrow \mathrm{Si}+2 \mathrm{H}_{2} ; \\
\mathrm{SiH}_{4}+2 \mathrm{H}_{2} \mathrm{O} \rightarrow \mathrm{SiO}_{2}+2 \mathrm{H}_{2} ; \\
\mathrm{SiOH}+\mathrm{SiOH} \rightarrow \mathrm{SiOSi}+\mathrm{H}_{2} \mathrm{O} ; \\
\mathrm{Si}+2 \mathrm{H}_{2} \mathrm{O} \rightarrow \mathrm{SiO}_{2}+2 \mathrm{H}_{2} ; \\
2 \mathrm{H}_{2}+\mathrm{SiF}_{4} \rightarrow \mathrm{Si}_{+}+4 \mathrm{HF} ; \\
4 \mathrm{HF}+2 \mathrm{SiO}_{2} \rightarrow \mathrm{H}_{4} \mathrm{SiO}_{4}+\mathrm{SiF}_{2}+\mathrm{F}_{2} ; \\
3 \mathrm{SiF}+2 \mathrm{H}_{2} \mathrm{O} \rightarrow 2 \mathrm{H}_{2} \mathrm{SiF}_{6}+\mathrm{SiO}_{2} ; \\
\left(\mathrm{SiO}_{2}\right)_{\mathrm{x}-1}+\mathrm{H}_{4} \mathrm{SiO}_{4} \rightarrow\left(\mathrm{SiO}_{2}\right)_{\mathrm{x}}+2 \mathrm{H}_{2} \mathrm{O} ; \\
\mathrm{Si}+\mathrm{SiF}_{\rightarrow} \rightarrow \mathrm{SiF}+\mathrm{SiF}_{2} \\
\mathrm{Si}+\mathrm{SiF}_{4} \rightarrow 2 \mathrm{SiF}_{2} .
\end{gathered}
$$

It is seen from spectroscopic data, that by low temperature conditions many molecular configurations are stable. The silane molecule dissociates and $\mathrm{SiH}$ or $\mathrm{SiH}_{2}$ fragments are chemisorbed on substrate. The dissociation of $\mathrm{SiF}_{4}$ molecule causes the appearance of fluorine on the film surface, but the hydrogen atoms react with fluorine and excess fluorine is removed. However, by increase of $\mathrm{SiF}_{4}$ flow rate, the $\mathrm{SiF}$ and $\mathrm{SiF}_{2}$ bonding are incorporated in silicon net on the film's surface. The hydrogen is incorporated in fluorinated silicon net by low temperature $\left(120^{\circ} \mathrm{C}\right)$ and reacts with oxygen to produce the $\mathrm{H}_{4} \mathrm{SiO}_{4}$ assembly.By increasing in temperature of deposition the molecular creation and dissociation rates increase. The ortho-silicon acid $\mathrm{H}_{4} \mathrm{SiO}_{4}$ dissociation into several molecular fragments with various quantities of hydrogen and oxygen atoms. The same situation is occurred for the silicon hydrogen fluorine acid, $\mathrm{H}_{2} \mathrm{SiF}_{6}$. In addition, it is necessary to note that the $\left(\mathrm{SiO}_{2}\right)_{\mathrm{x}}$ polymeric configuration, as gel, are appeared in silicon net. It is easily transformed into $\mathrm{SiO}_{\mathrm{x}}$ material for nanoscale sizes.

\subsection{Structural and Chemical Properties of Films}

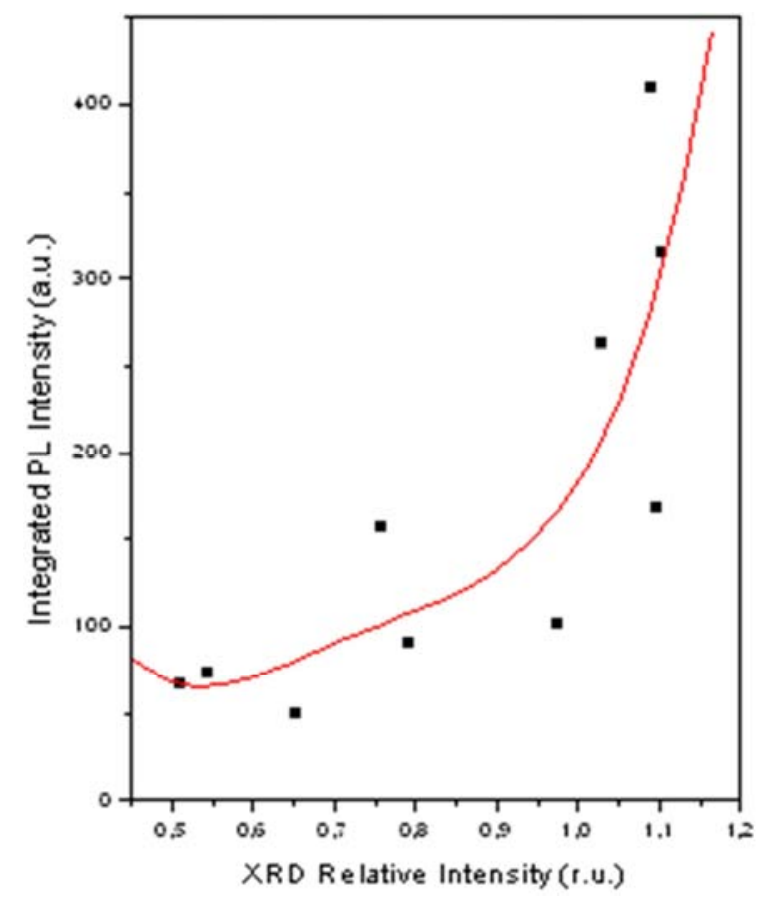

Figure 1. Nonlinear relation between the PL integrated intensity and XRD relative intensity of signal by the angle of diffraction $28^{\circ}$ for silicon nanocrystalline film.

Correlation between photoluminescence (PL) Integrated Intensity and XRD relative intensity of signal by the angle of diffraction $28^{\circ}$ for silicon nanocrystalline film is illustrated in the Figure 1. There is a nonlinear relation between both PL data and XRD data. It is assumed, that the expression for the intensity of PL can be written as

$$
I_{P L}(\delta) \propto \frac{A}{\delta^{2}}=A B^{2} ;
$$

but the triangular approximation for XRD integral intensity show accordingly by means ofScherer formula that

$$
I_{X R D} \propto \frac{\mathrm{H} \cdot \mathrm{C}}{\delta}=H B .
$$

$H$ is a magnitude of XRD peak. It is found that the PL peak energy changes in a strong correlation with the $<\delta>$ value. In addition to such a size effect on the PL response, the energy band gap may increase as the films are hydrogenated. Furthermore, the hydrogenation is also expected to terminate Si dangling bonds which act as non-radiative recombination centers. Spectral characteristics of Gauss-distributed oscillators with their eigen-frequiencies. Integrated PL Intensity can be described by using following way 


$$
I_{\Sigma P L}=\int_{0}^{\infty} I(\mathrm{w}) d \mathrm{w}
$$

is the integral intensity of PL nanocrystals. For amount of nanocrystals emitted light with fixed frequency we can write,

$$
N(\mathrm{w})=\int_{0}^{\infty} f(\delta) \vartheta(\delta) \exp (-i \mathrm{w} \delta) d \delta
$$

and the intensity of radiation from nanocrystals with definite size is following

$$
I(\delta)=\frac{1}{2 \pi} \int_{0}^{\infty} N(\mathrm{w}) \exp (i \mathrm{w} \delta) d \mathrm{w} .
$$

It is studied the relationship between the optical and structural properties of poly-Si films prepared at low temperature PECVD. The experimental investigations by Raman scattering spectroscopy of silicon films were carried on by means of spectroscopic technique JASCO NRS-1000 (Japan).The correlation among the PL response, structural and other optical properties (Raman scattering and IR absorption) of poly-Si films with nanocrystals was studied changing the of hydrogen flow rate (from $10 \mathrm{sccm}$ to $46 \mathrm{sccm}$ ) under $\left[\mathrm{SiF}_{4} / \mathrm{He}\right]$ $=2.5 \mathrm{sccm}$. It is shown in Figure 2 that there is a strong correlation among the PL intensity, Raman intensity, and the average grain size of crystallites and density of hydrogen bonds for films with temperatureat $300^{\circ} \mathrm{C}$.The average grain size of crystallites with preferential (111) or (220) orientations decreases by increasing the hydrogen flow rate. Furthermore, the absorption properties and the density of $\mathrm{Si}-\mathrm{H}$ and $\mathrm{Si}-\mathrm{F}$ related bonds were investigated by varying the $\mathrm{H}_{2}$ and $\mathrm{SiF}_{4}$ flow rates. As stated above, the PL spectra may be controlled by the shape of the size distribution. In this case, if we suppose the state-to-state spontaneous transition probability, the PL intensity $I(E)$ may be expressed as

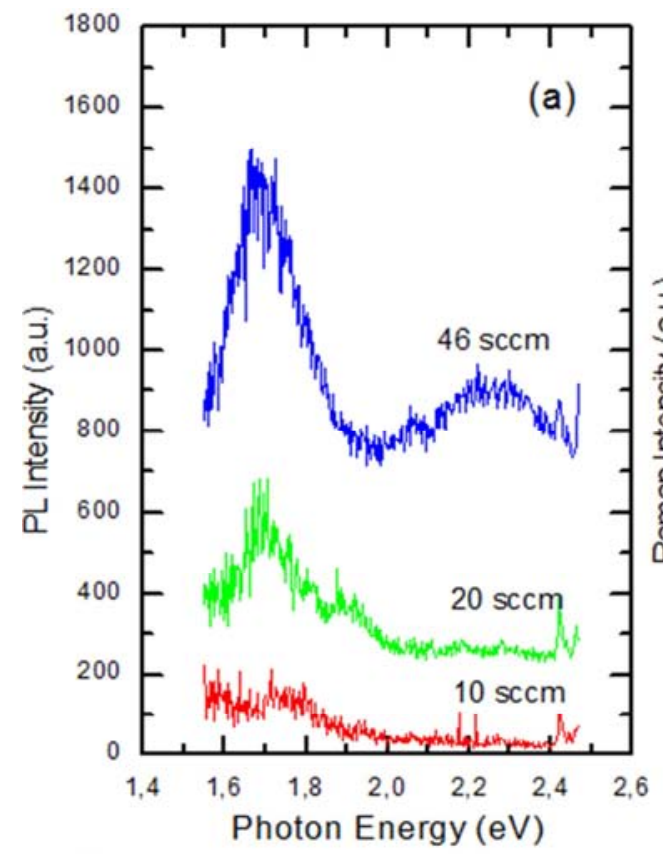

$$
I(E) \sim W_{i j} f(\delta),
$$

where $W_{i j}=\frac{4 e^{2} E n}{3 m^{2} h^{2} c^{3}}|<i| p|j>|^{2}$, e and $\mathrm{m}$ are the charge and mass of electron, respectively, Eisthe transition energy, $p$ is the transition dipole momentum operator, $n$ is the refractive index, and

$$
f(\delta)=\frac{1}{2 \pi \sigma} \exp \left(-\frac{1}{2}\left(\frac{\delta-<\delta>}{\sigma}\right)^{2}\right)
$$

is the Gaussian distribution of grain sizes, $\sigma$ is the standard deviation.

Figure 2 shows the PL spectrum of nc-Si film in the energy range from $1.5 \mathrm{eV}$ to $2.5 \mathrm{eV}$. The spectrum consists of high energy part with smooth peak around 2.2-2.4 eV, and low energy part with sharp peak around $1.8 \mathrm{eV}$. The very sharp peaks in the higher energetic partcorrespond to the molecular spectra of oxygen on the silicon surface. The low energy spectral peak can be divided into two closed peaks. The first spectral peak with maximum around $1.80 \mathrm{eV}$ is an optical response of a-Si:H interface media, but the second- around $1.85 \mathrm{eV}$ is due to the band-to-band transitions from the tail of conductive band of silicon nanocrystals. The smooth peak around $2.2-2.4 \mathrm{eV}$ is caused by the optical transitions from the defect levels made by oxygen incorporation. We suppose that there is oxygen-related defect levels inside band gap with energy position by direct optical transition in silicon E$2.2 \mathrm{eV}$, where $\mathrm{E}=3.37-3.4 \mathrm{eV}$ for point $\Gamma$ of the Brillouin zone. In this case we assume the two photon optical transition by PL process with photon energies equal to $2.2 \mathrm{eV}$ and $1.2 \mathrm{eV}$, respectively. The $\Gamma-\mathrm{X}$ indirect transitions are possible, too. Figure 3 illustrates the FT IR absorption spectra for these three films which other spectral characteristics were shown in Figure 2. There are spectral lines around $1100 \mathrm{~cm}^{-1}$ that correspond of Si-O-Si bridges.

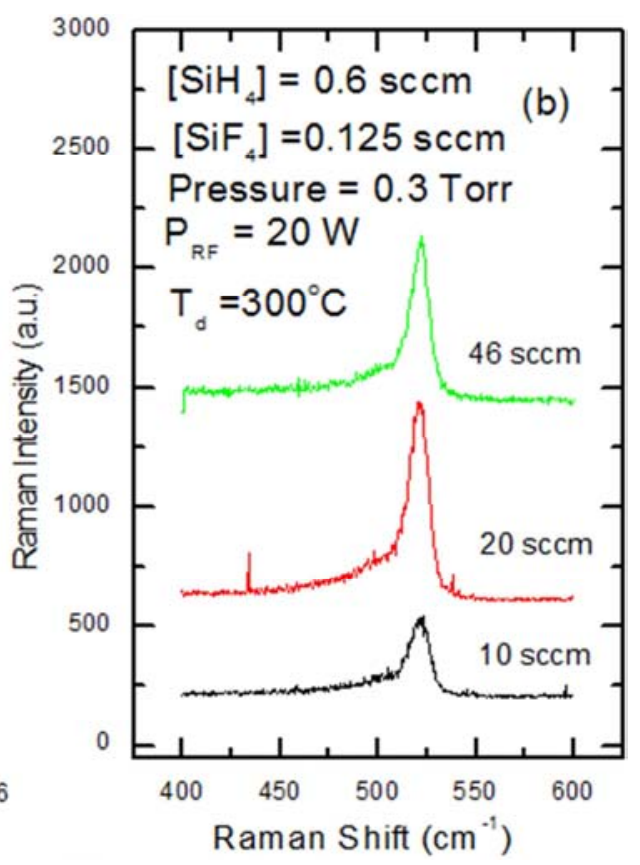



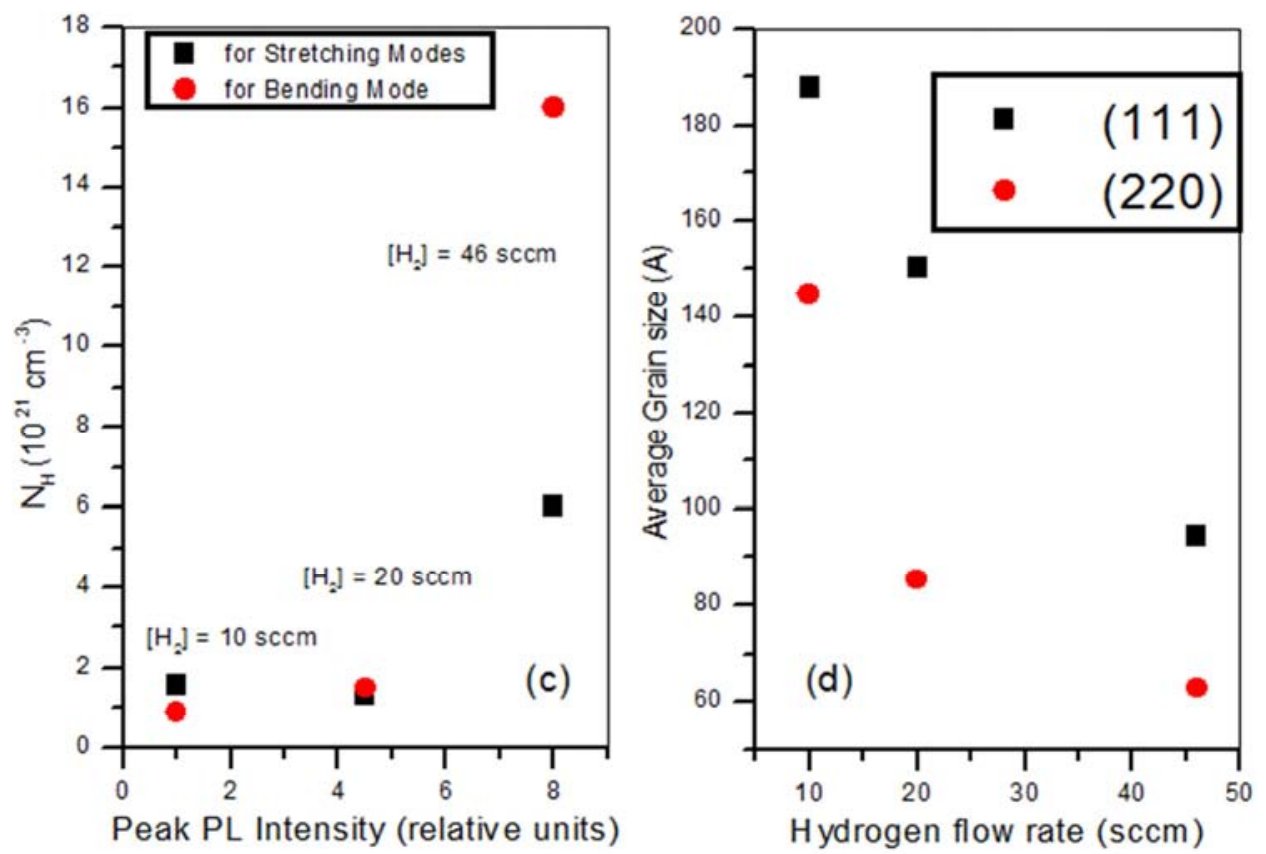

Figure 2. PL and Raman spectra, concentration of hydrogen bonds, and average grain size (estimated from XRD data) for polycrystalline silicon films.
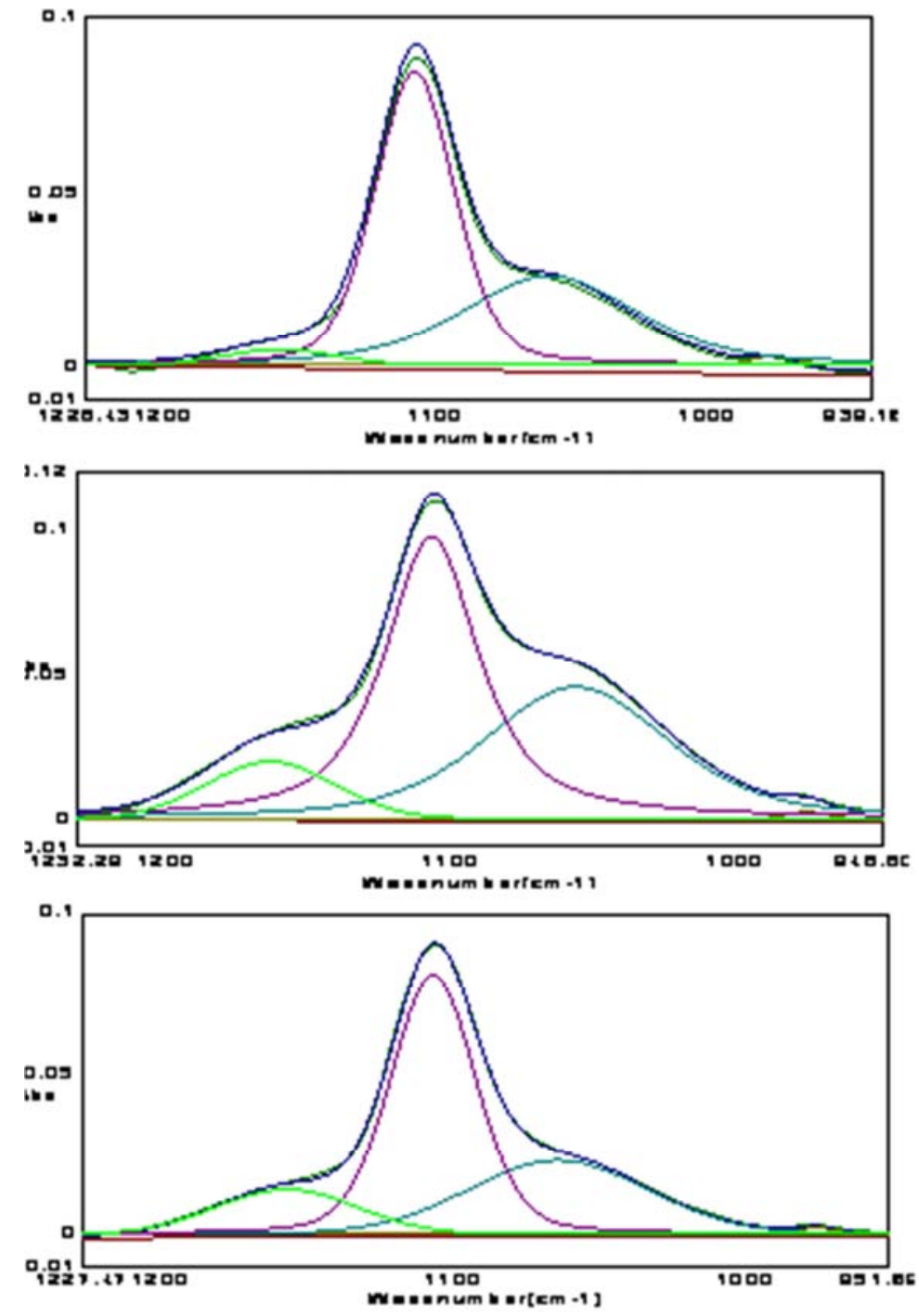

Figure 3. Fourier-Transformed Infrared spectroscopy data for silicon films. 

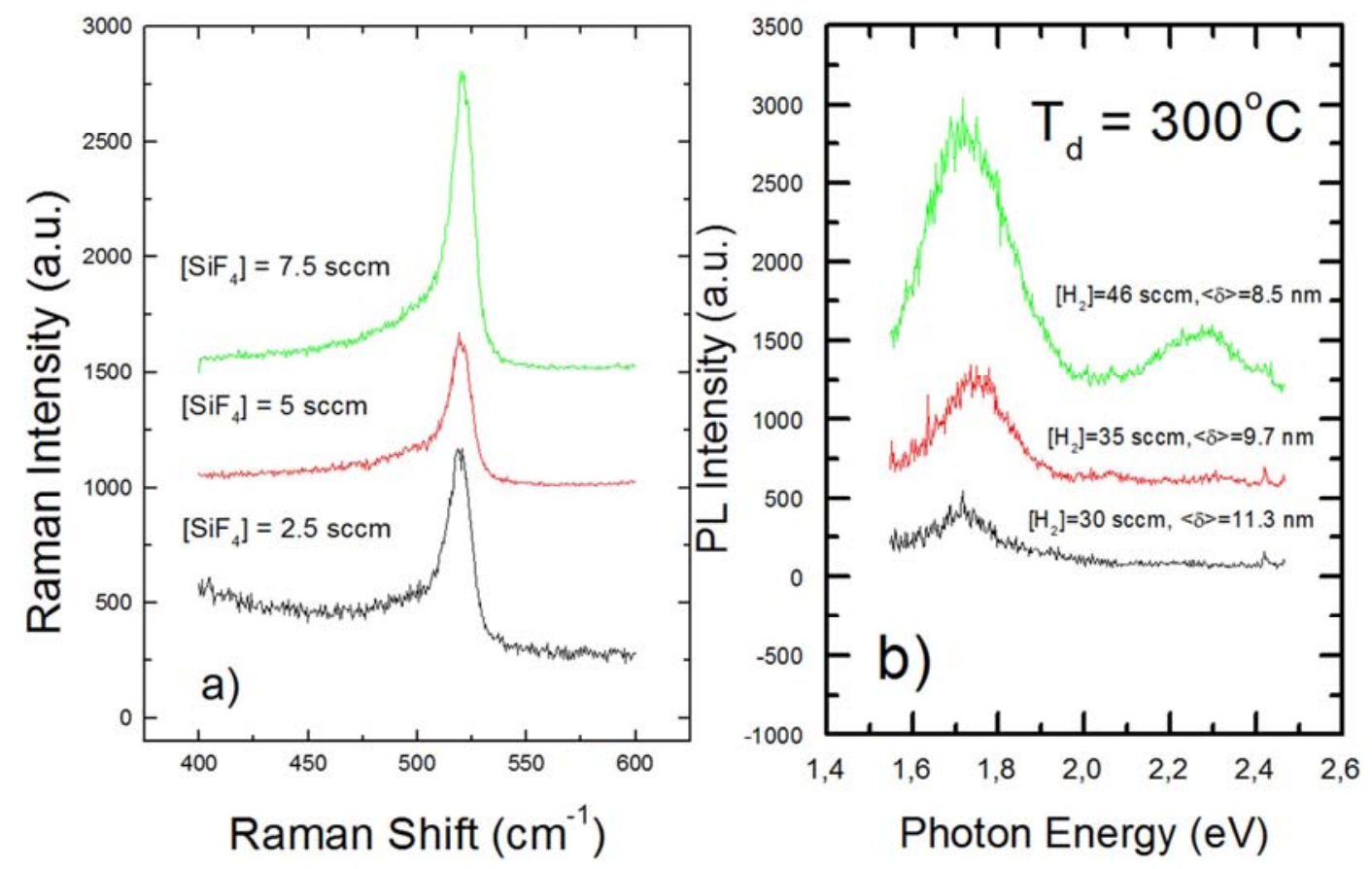

Figure 4. Raman (a) and PL (b) spectra for fluorinated nanocrystalline silicon films.
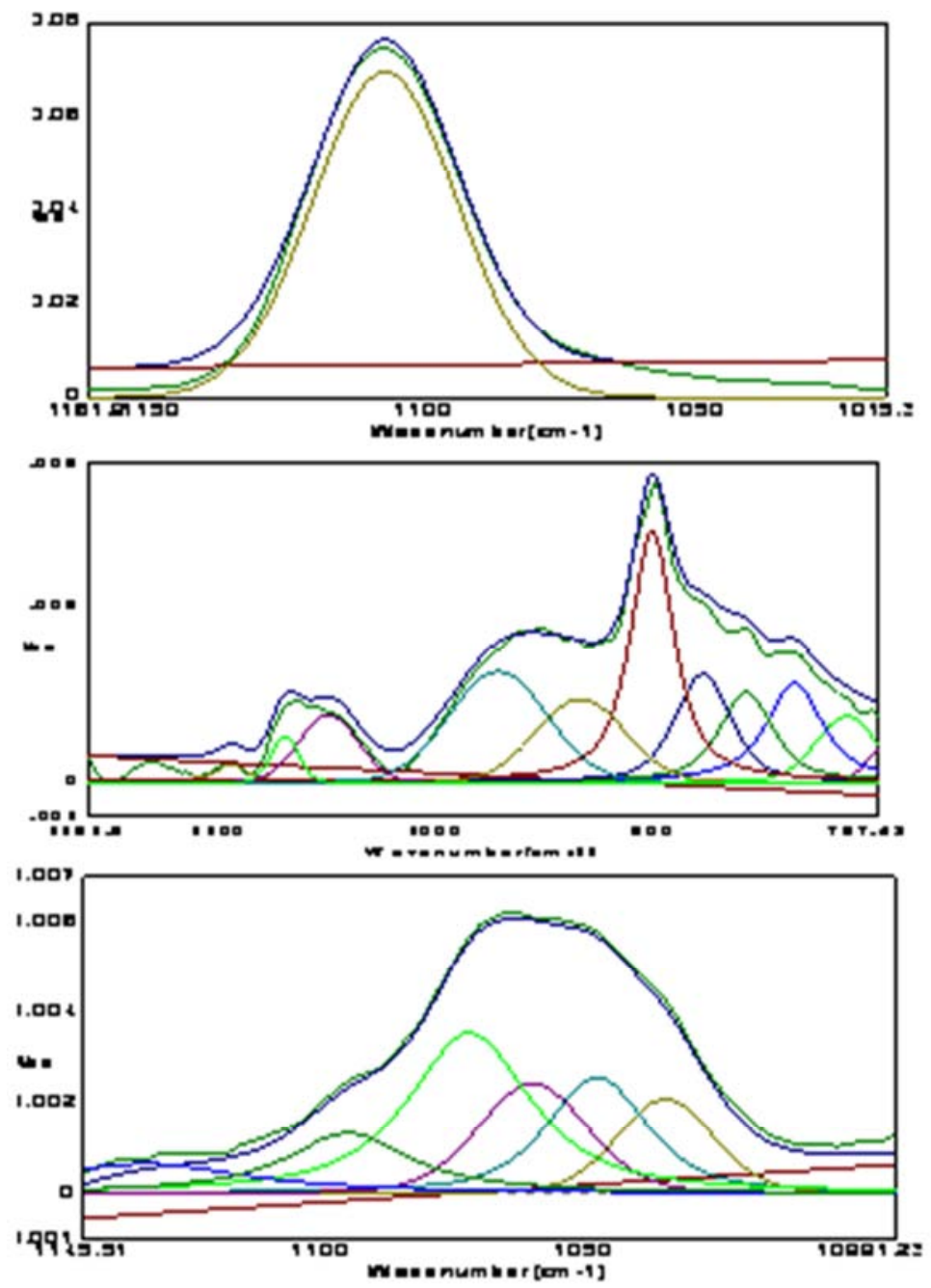

Figure 5. Fourier-Transformed Infrared spectroscopy data for fluorinated silicon films. 
For the PL intensity we can use the formula (6)

$$
I \approx \frac{1}{2 \alpha} I_{L} W_{i j} \rho D^{2} N_{n c-S i}
$$

Where $\alpha$ is coefficient of absorption of silicon $\left(3 * 10^{4} \mathrm{~cm}^{-}\right.$ $\left.{ }^{1}\right), D$ is diameter of laser beam $\left(10^{-2} \mathrm{~cm}^{2}\right)$, pis a crystalline volume fraction (around 0.7).

Figure 4 shows the Raman and PL spectra for the nanocrystalline silicon films prepared by using silicon tetra fluoride gas. It is clear, that there is strong spectral peak at the 1.6-1.8 eV the magnitude of which increases by increasing of films hydrogenation. Figure 5 shows the FT IR absorption spectra which related to the spectral data in Figure $4 \mathrm{~b}$, respectively. It is seen, that the silicon oxides are disappear but silicon fluorides are creates by fluorination of films.

\section{Atomic-Force Microscopic Photos of Silicon Films and Film Morphology}

Atomic-force microscopic (AFM) measurements were carried on by means of Scientific Park Instruments (USA) technique. Surface roughness can be evaluated by means of average roughness parameter (or mean surface level) and root means square (RMS) parameter (see Figure 6). Both parameters can be calculated from integral over length

$$
\begin{aligned}
& R=\frac{1}{L} \int_{0}^{L} \mid r(x) d x ; \\
& R M S=\sqrt{\frac{1}{L} \int_{0}^{L} r^{2}(x) d x .}
\end{aligned}
$$

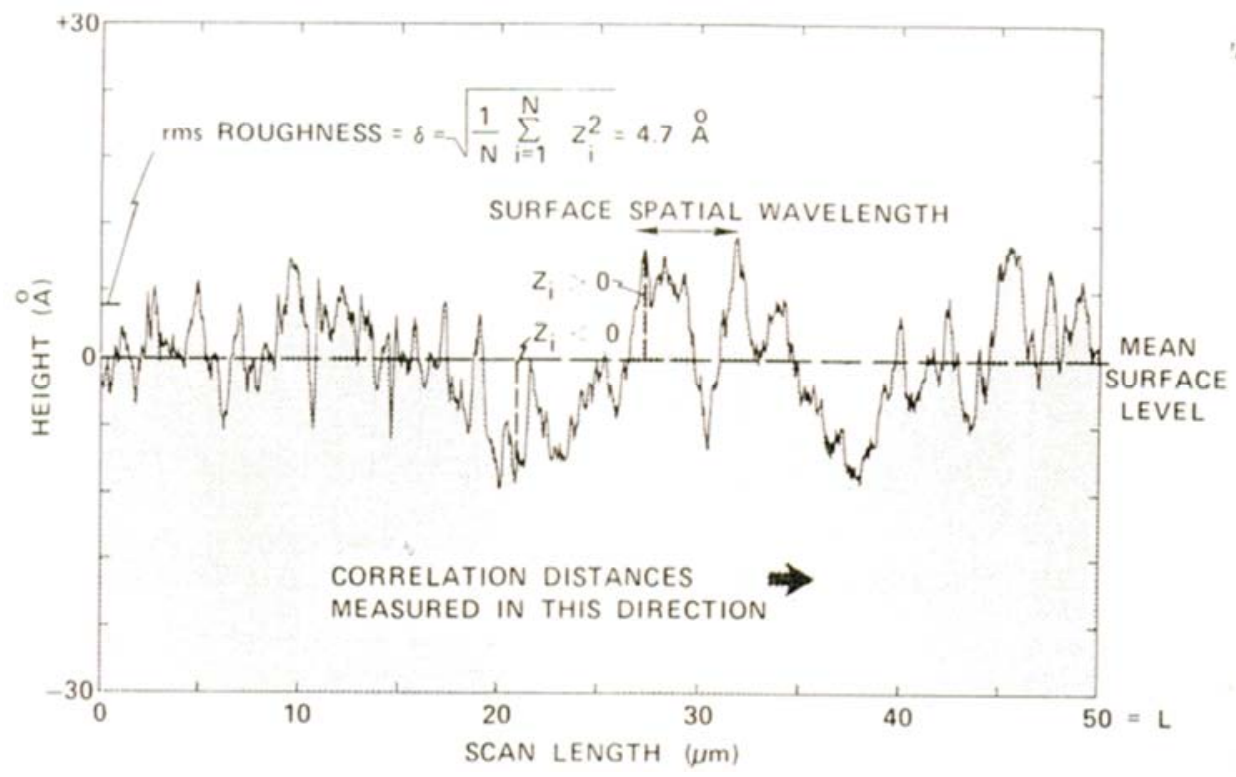

Figure 6. Roughness parameters for surface of film [5].

The typical values for nanocrystalline films deposited by using different facility are shown in the Table 1. It is seen, that the RMS parameters for films deposited by using APEX facility are in the range of 2 to $5 \mathrm{~nm}$ [5], and from $1 \mathrm{~nm}$ to 7 $\mathrm{nm}$ for ordinary CVD reactor. However, the film structural parameters such as thickness of film, spatial homogeneity of crystallinity, the density of point defects can be significant differ from each other. It is dramatic to produce the semiconductor devices with high quality.

It is seen in Table 1 , that the R and RMS parameters are less than $4.5 \mathrm{~nm}$ and $3.5 \mathrm{~nm}$, respectively for the films which were analyzed by AFM method.

Table 1. Roughness parameters for surface of nanocrystalline silicon film.

\begin{tabular}{lllll}
\hline Line & Height $\AA$ & Distance, $\boldsymbol{\mu m}$ & Rms, $\AA$ & Average, $\AA$ \\
\hline A & 43.1 & 0.651 & 32.2 & 21.8 \\
B & 11.5 & 0.651 & 43.1 & 33.5 \\
C & 23.9 & 0.651 & 25.3 & 19.4 \\
D & 71.8 & 0.651 & 30.2 & 22.8 \\
E & 45.0 & 0.651 & 26.9 & 20.4 \\
\hline
\end{tabular}

\begin{tabular}{lllll}
\hline Line & Height $\AA$ & Distance, $\boldsymbol{\mu m}$ & Rms, $\boldsymbol{\AA}$ & Average, $\boldsymbol{\AA}$ \\
\hline $\mathrm{F}$ & 18.2 & 0.651 & 21.6 & 16.8 \\
$\mathrm{G}$ & 7.66 & 0.651 & 32.7 & 26.2 \\
\hline
\end{tabular}

Figure 7 illustrates the Atomic-force spectroscopic data for the silicon films the spectroscopic data of which were shown in Figure $4 \mathrm{~b}$. It is seen, that the increasing of hydrogen concentration in film with its fluorination results in structural changes from nanocrystallites (see Figure $7 \mathrm{a}$ and Figure $7 \mathrm{~b}$ ) to monocrystal structures as it is seen in Figure $7 \mathrm{c}$.

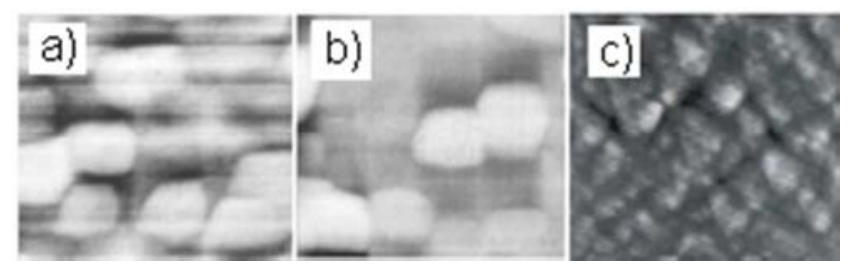

Figure 7. Atomic-force spectroscopic data for the silicon films the spectroscopic data of which were shown in Figure 4. 
Silicon crystalline film with orientation (111) was prepared at the first time on glass substrate by using the plasmaenhanced chemical vapor deposition as it is seen in Figure 7c. The structural changes were controlled by using the etching and growing processes at the low temperature. The surface/volume ratio was varied from0.01 $1 / \AA ̊$ to $0.041 / \AA$. The homogeneity of crystal phase was also tested by using Raman mapping measurements and nonlinear laser spectroscopy [6]. The concentration of hydrogen was $4 \%$, according to the FT IR data.

The surface roughness and morphology parameters are comparableto the morphology parameters (RMS is varied from $2 \mathrm{~nm}$ to $4 \mathrm{~nm}$ ) of thin (thickness less than $100 \mathrm{~nm}$ ) amorphous silicon films with nanocrystals (average grain size less than $3 \mathrm{~nm}$, crystalline volume fraction is lower than $20 \%$ ) deposited by using APEX facility by means of silane gas [flow rate in range of 20 to $100 \mathrm{sccm}$ ] diluted by hydrogen [which is varied from $2000 \mathrm{sccm}$ to $8000 \mathrm{sccm}$ ] with great values of RF power (from $400 \mathrm{~W}$ to $600 \mathrm{~W}$ ) and the same temperature of deposition [7] and show the better results than these.
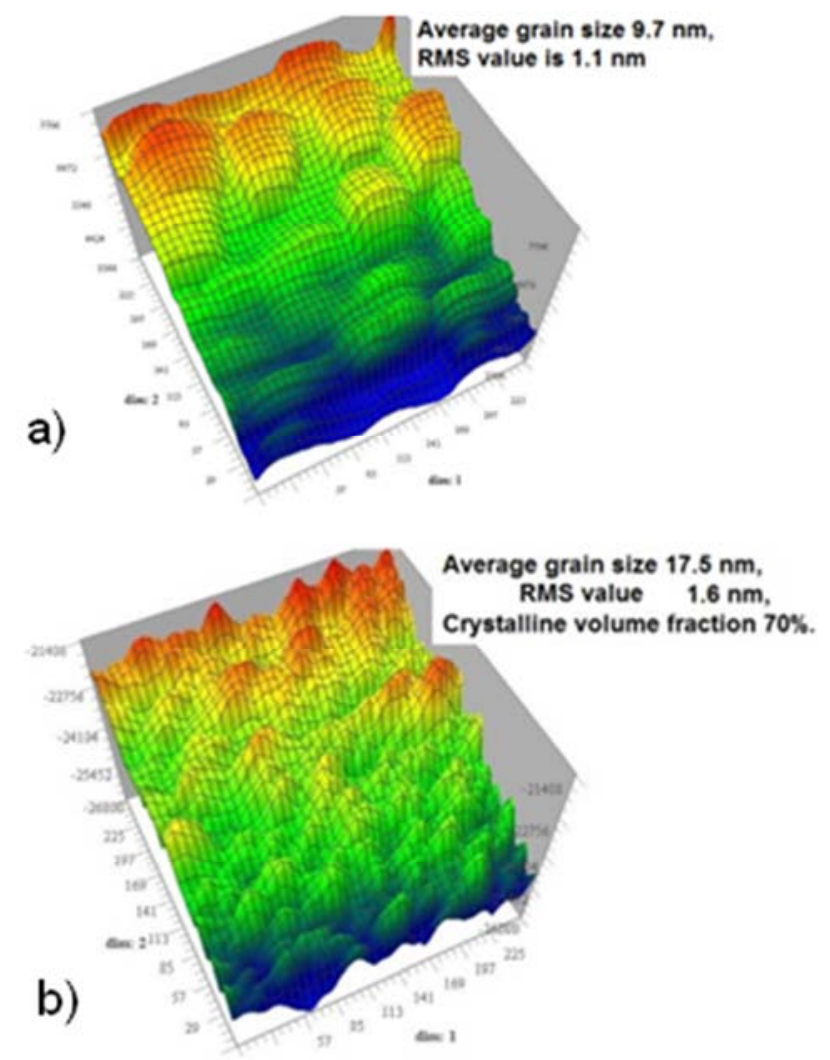

Figure 8. Atomic-force microscopic data to estimate the RMS values and other morphological parameters for nanocrystalline films with determined average grain sizes according to the XRD data prepared by: low (a) and high (b) $\mathrm{SiF}_{4}$ flow rates in electrochemical reactor.

Figure 8 illustrates the changes in morphology of films by their significant fluorination. It is seen in Figure 8a that the silicon nanocrystal grains have the rectangular shape and great area of $\mathrm{Si}(111)$ crystal orientation. By adding the silicon tetra fluoride in electrochemical reactor the etching of nanocrystals by the temperature of substrate $300{ }^{\circ} \mathrm{C}$ is significant and there are numerous sharp hills on the film surface.

\section{Discussion}

We observed the dependence of PL peak energy and integrated PL intensity on the sizes of crystallites for films prepared by changing the deposition conditions. The PL response of poly-Si films is results of the presence of nanocrystallites and quantum confinement effects in them. The luminescent properties of films are likely to depend on the size distribution of crystallites, along with an effect of hydrogen termination on the surface of the crystallites.

The amount of nanocrystals is proportional to the area limited by the two curves in Figure 9 and can be written as [8]
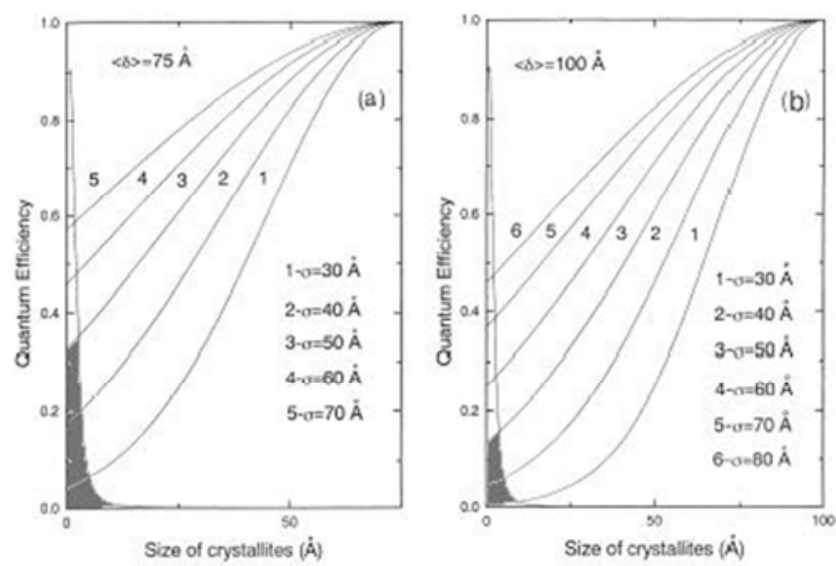

Figure 9. Quantum emission function and various size distribution functions for the determination the number of nanocrystals which are responsible for $P L$.

$$
N_{n c-S i}=\frac{1}{\sqrt{2 \pi} \sigma} \int_{0}^{x_{c}} \exp \left(-\frac{1}{2} \frac{(x-\mu)^{2}}{\sigma^{2}}\right) d x+\int_{x_{c}}^{\infty} \frac{d x}{1+\gamma x^{3}}
$$

Where $x_{c}$ is a critical nanocrystal size value that can be found by using the graphics in Figure 9 by the following way

$$
\frac{1}{\sqrt{2 \pi} \sigma} \exp \left(-\frac{1}{2} \frac{(x-\mu)^{2}}{\sigma^{2}}\right)=\frac{1}{1+\gamma x^{3}}
$$

where the $x_{c}$ is a root of equation. By the simple way to decay the exponential function this equation can be transformed into the form:

$$
A x_{c}^{3}+B x_{c}^{2}+C x_{c}+D=0
$$

It is supposed, that the large quantity of nanocrystals is Gauss distributed in their sizes. PL peak position for silicon films can be estimated by using experimental data $E_{P L \max }-E_{b g}=\frac{A}{\langle\delta\rangle^{1 / 3}}$. The nanocrystals with lowest 
sizes result in most efficient PL. Accordingly, the quantity of emitted hydrogenated nanocrystals by band-to-band radiative transitions is important to estimate the PL linear optical response [9]. The surface morphology of film play great role only in Raleye scattering phenomena, but for the Raman and PL emission can be neglected, because in these cases there is a great role of dominant volume effect. The hydrogenation of silicon nanocrystals for these films is most important by the PL emission, despite the role of oxide related point defects [10].

In conclusion, I studied the photoluminescence of silicon nanocrystalline hydrogenated films, their chemical and structural properties. Technological investigations show the possibility the manufacturing of fully crystallized silicon film deposited on glass substrate. It is possible due to the combination of low temperature deposition conditions, film etching by silicon fluorides during the deposition, hydrogenation of film. It is established that PL depends on mainly crystalline silicon fraction. Mechanism of PL emission by small silicon nanocrystals was proposed.

\section{References}

[1] B. Sweryda-Krawiec, T. Cassagneau, J. H. Fendler, J.Phys. Chem.B, Vol.103, 1999, pp. 9524-9529.

[2] L. Rego, S. G. Abuabara, S. Batista, The Journal of Chemical Physics, Vol.122, 2005, p.154709.

[3] P. Wu, D. Lin, Growth mode in $\mathrm{Si}(100)$ epitaxy by low temperature chemical vapor deposition, Physical Review B, Vol. 57, No.19, 1998, pp. 12421-12427.
[4] N. Wang, Y. Tang, Y. Zhang, C. Lee, S. Lee, Nucleation and growth of Si nanowires from silicon oxide, Physical Review B, Vol.58, No.24, 1998, pp. R16022-16026.

[5] J. Bennet and L. Mattson, Introduction to surface roughness and scattering, Optical Society of America, Washington, D.C., 1989, pp.38-39.

[6] D. Milovzorov, Surface States Silicon Crystalline Films Detected by Nonlinear Laser Spectroscopy, Norvegian Journal of Development of the International Science, 6, 2017, pp.7482.

[7] D. Milovzorov, K. B. Kim, M. Lisachenko, J. W. Seo, K. Y. Lee, and H. K. Chung, Microcrystalline Silicon for Thin Film Transistor, Proceedings of the 5th International Meeting on Information Display (IMID), Seoul, South Korea, July 19, Vol. II, 2005, pp.1320-1322.

[8] D. Milovzorov, T. Inokuma, Y. Kurata, and S. Hasegawa, Relationship Between Structural and Optical Properties in Polycrystalline Silicon Films Prepared at Low Temperature by Plasma-Enhanced Chemical Vapor Deposition, Journal of the Electrochemical Society, 145, 1998, 3615-3620.

[9] D. E. Milovzorov, A. M. Ali, T. Inokuma, Y. Kurata, T. Suzuki and S. Hasegawa, Optical properties of silicon nanocrystallites in polycrystalline silicon films prepared at low temperature by plasma-enhanced chemical vapor deposition, Thin Solid Films, Vol.382 No1\&2, 2001, pp.47-55.

[10] D. Milovzorov, Laser Assisted Photoprocesses in Nanostructured Silicon Films, Advanced Nanomaterials and Technologies for Energy Sector-NanoEnergy, 1, 2017, pp.2133 